\title{
ProductNews
}

\section{IR Lenses}

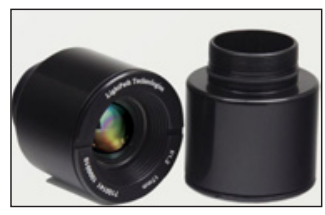

ISP Optics provides a variety of aspheric IR lenses capable of achieving maximum performance for IR objective lenses. Infrared objective lenses open a new door of applications when compared to visible objective lenses. When considering IR objective lenses, it is important to be efficient with the lens selection, as smaller focal points allow for better precision, without compromising transmission. ISP offers off-the-shelf products, as well as build-to-print manufacturing services to help with custom IR optical components.

LightPath Technologies, Inc.

www.lightpath.com

\section{Vented Hood Table Top Workstation Model 24000}

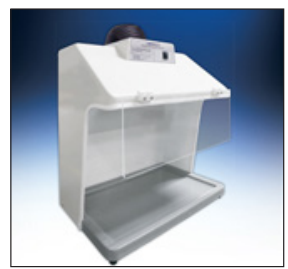

Typical uses include histology, microprocessor, venting for hot plates, microscope stations, student workstations, sample weighing stations, and handling pharmaceuticals. Constructed of chemical resistant, lightweight advanced composites, it can be easily moved as procedures or workflow change. Dimensions are 24 " wide $\times$ 15 " deep $\times 24$ " high. Molded chemical-resistant work surface is recessed to contain spillage, and a three-inch diameter outlet collar is provided for duct connection.

HEMCO Corporation

www.HEMCOcorp.com/ductless.htm

Sona, The World's Most Sensitive Back-Illuminated sCMOS Camera

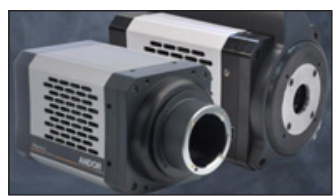

The new Sona back-illuminated sCMOS camera, deep-cooled to $-45^{\circ} \mathrm{C}$, has been designed for fluorescence microscopy, optimizing signal to noise under reduced illumination conditions, thus preserving living cells during extended measurement periods. With the largest field of view from a back-illuminated sCMOS, Sona is perfect for applications requiring maximum information content, allowing large fields of cells, whole embryos, or tissue cultures to be captured with absolute clarity and maximum throughput.

\section{Andor Technology}

andor.oxinst.com

\section{$532 \mathrm{~nm}$ Accessories for FERGIE® Spectrographs}

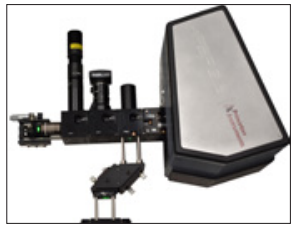

Princeton Instruments announced the immediate availability of $532 \mathrm{~nm}$ Raman accessories for its FERGIE spectrometer product line. FERGIE is a fully integrated, aberration-free spectrograph with a built-in, low-noise, cooled detector. FERGIE provides the high sensitivity and low noise needed to address a wide range of spectroscopy applications. Its ecosystem of easy-to-use accessories, which includes everything from lasers, fiberoptics, and calibration sources to the ingeniously designed family of light-coupling FERGIE CUBES.

Princeton Instruments

www.fergiespec.com

New 9 and 12 MP Industrial Cameras with GigE ix Industrial Ethernet Interface

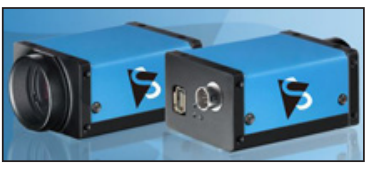

The Imaging Source introduced four new industrial cameras featuring the latest, large-format Sony Pregius CMOS sensors and the robust ix Industrial $l^{\triangleright}$ ethernet interface. The " 38 series" color and monochrome cameras are equipped with Sony's costeffective IMX267 and IMX304 sensors, which offer high resolution (8.8 $\mathrm{MP}$ at $13 \mathrm{fps}$ and $12.3 \mathrm{MP}$ at $9 \mathrm{fps}$, respectively) in addition to high dynamic range with low noise and brilliant color reproduction.

The Imaging Source

www.theimagingsource.com

\section{UniFlow Specialty Fume Hoods}

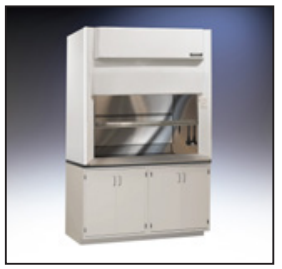

These hoods are constructed in a variety of material options: type 316 stainless steel for perchloric acid, PVC for perchloric acid, HDPE for acid digestion, polypropylene for trace metals, and type 304 stainless steel for radioisotope procedures. All specialty hoods feature welded one-piece interiors including the worksurface. Perchloric acid hoods also have a welded-in rear drain trough for washdown. All Specialty Fume Hoods will have vapor-proof or explosion-proof lighting, front airfoil, rear baffle, and duct connection collars.

HEMCO Corporation

www.hemcocorp.com

\section{New ace U Models with 20 MP Resolution}

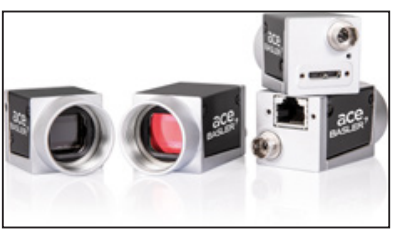

Camera manufacturer Basler presents four new ace $U$ models with the rolling shutter CMOS sensor IMX183 from the Sony Exmor R series. The cameras offer a resolution of 20 megapixels with a sensor size of $1 "$ and a compact design with a $29 \mathrm{~mm} \times 29 \mathrm{~mm}$ footprint. This combination makes the four new ace $U$ models particularly attractive for inspection of the finest structures, such as in PCB or display production.

\section{Basler AG}

www.baslerweb.com

\section{TESCAN Launch S9000X - Their Flagship Xe Plasma}

\section{FIB-SEM}

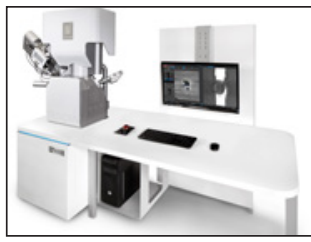
50 times faster than conventional gallium FIBs. Furthermore, the use of Xe helps to overcome some of the drawbacks of using gallium, including amorphization, which can lead to incorrect analyses.

AXT Pty Ltd

www.axt.com.au 
Pfeiffer Vacuum Presents New Two-Stage Rotary Vane Pump Pascal 2021 HW

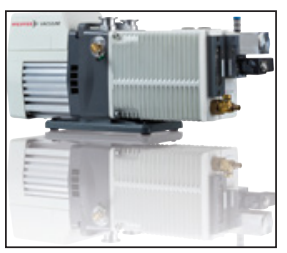

Pfeiffer Vacuum presents a two-stage rotary vane pump with the highest vapor capacity in its class. It is optimally suited for all applications where water vapor is expected, such as low-temperature sterilization and drying. An optimized design and gas ballast system allows it to pump large volumes of vapor without condensation inside the pump. The Pascal $2021 \mathrm{HW}$ also resists aggressive chemicals such as hydrogen peroxide.

Pfeiffer Vacuum GmbH

www.pfeiffer-vacuum.com

Four New ace U Models with the IMX287 and IMX273

Sensors from Sony

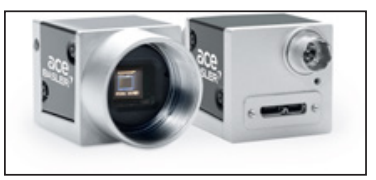

Four new ace U USB 3.0 models from Basler feature resolutions of VGA and 1.6 megapixels and have now moved into series production. They are equipped with the IMX287 and IMX273 sensors from Sony's Pregius series and deliver up to 525 frames per second. The IMX273 sensor offers outstanding Pregius image quality, high speeds, and low noise. The IMX287 sensor has twice the pixel size with an edge length of $6.9 \mu \mathrm{m}$.

Basler AG

www.baslerweb.com

\section{Microflow III Class 1 Ductless Workstation}

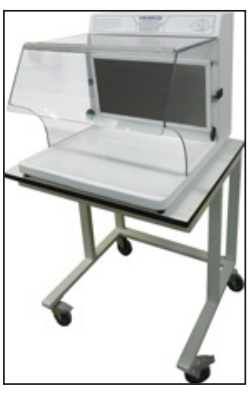

The MicroFlow III is a Class 1 ductless carbon-filtered workstation equipped with particle pre-filter and activated carbon filtration, ideal for fumes, odors, and non-hazardous chemical vapors. It is completely self-contained with integral recessed work surface to contain spills. A viewing sash surrounds the work area and can be conformed for use with a microscope variable-speed fan control, which allows for high-speed 100f/m airflow through the sash opening, or medium and low flow for sensitive operations.

Hemco Corp.

www.HEMCOcorp.com

\section{X-Cite $₫$ XYLIS, Brilliance Across the Spectrum}

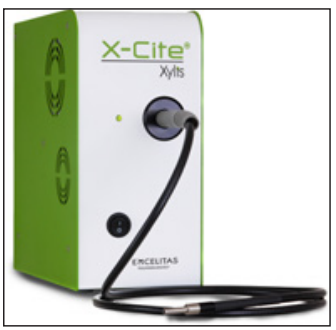

$\mathrm{X}$-Cite XYLIS is a true arc lamp replacement for routine and advanced fluorescence imaging applications. It has the broadest spectrum available in a white light LED for fluorescence microscopy and rivals traditional arc lamps for brightness, making it ideal for both compound and stereomicroscopes. Incorporating its patented and award-winning LaserLED Hybrid Drive ${ }^{\varpi}$ technology to overcome the LED green gap, X-Cite ${ }^{\oplus}$ XYIS makes it possible to have the benefits of LEDs without compromising on flexibility or performance.

Excelitas Technologies Corp.

www.excelitas.com/x-cite
JAl's Newest Spark Series 12.4-Megapixel Camera Delivers $64.6 \mathrm{fps}$

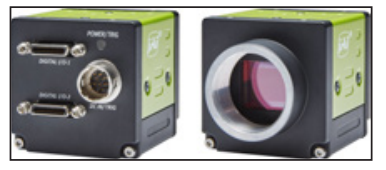

JAI introduced the SP-12400-PMCL, a new 12.4-megapixel camera in its Spark Series of high-resolution area scan cameras. Two models are offered: a monochrome model (SP12400M-PMCL) and a color model (SP-12400C-PMCL). The IMX253 is the faster "cousin" of the IMX304 imager featured in the SP12401-PGE (GigE Vision) model. Both imagers feature 3.45-micron pixels and a dark noise rating of less than 6 electrons for excellent image quality even under low light conditions.

JAI Inc.

www.jai.com

EasyTau 2 - Measure and Analyze Spectroscopy Data in One Package

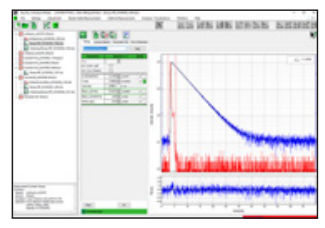

PicoQuant's new EasyTau 2 software package combines fully automated fluorescence spectra measurement with powerful and easy-touse data analysis. The newly released software package provides a fully integrated, comprehensive suite of fitting and analytical tools for time-resolved fluorescence spectroscopy applications. With this upgrade, EasyTau 2 becomes a one-stop solution for both acquiring and analyzing data with PicoQuant's fully automated, modular, and high-end FluoTime 300 spectrometer.

PicoQuant

www.picoquant.com

\section{Alpha3 Light Sheet Fluorescence Microscope}

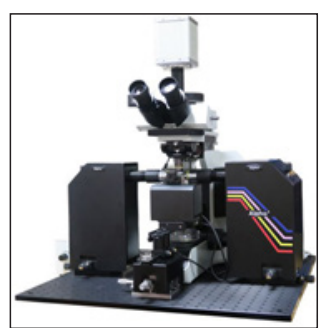

From in-vivo imaging to clearing, Alpha3 is a new generation of light sheet microscopy systems providing new quantitative and qualitative imaging capabilities. The Alpha3 modular configuration allows for easy adaptation to experimental constraints and enables fast and reproducible sample mounting. The advanced functionalities of sharp optical sectioning and smart scanning drastically alleviate spatial and temporal resolution constraints for 3D image acquisition and deliver high throughput 3D imaging from single cells to whole organisms.

PhaseView

www.phaseview.com

ASM Handbook, Volume 17: Nondestructive Evaluation of Materials

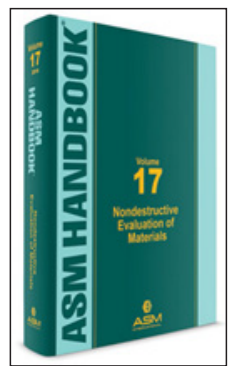

This ASM Handbook helps readers select, use, and interpret methods used to nondestructively test and analyze engineered products and assemblies. Digital technology is transforming the implementation of nondestructive evaluation (NDE) and is covered extensively. This volume is organized into seven divisions: an introduction to NDE, visual examination and optical inspection, thermal methods, sonic and ultrasonic techniques, radiography electromagnetic inspection, and articles that address selected applications of NDE to man-

ufactured products.

ASM International

www.asminternational.org/hbvol17 